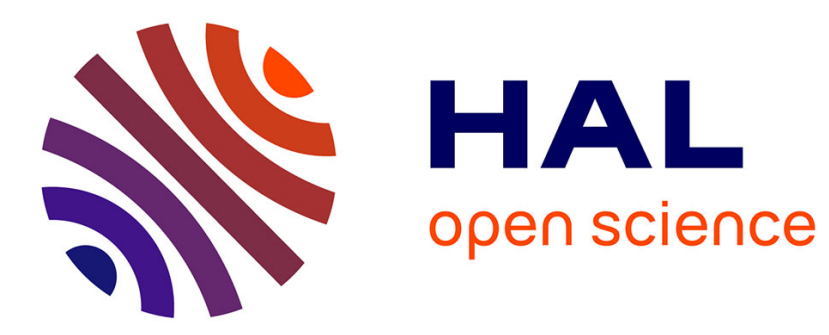

\title{
Optical index of fault tolerant routings in WDM networks
}

Stéphane Bessy, Clément Lepelletier

\section{To cite this version:}

Stéphane Bessy, Clément Lepelletier. Optical index of fault tolerant routings in WDM networks. Networks, 2010, 56 (2), pp.95-102. 10.1002/net.20351 . lirmm-00738222

\section{HAL Id: lirmm-00738222 \\ https://hal-lirmm.ccsd.cnrs.fr/lirmm-00738222}

Submitted on 3 Oct 2012

HAL is a multi-disciplinary open access archive for the deposit and dissemination of scientific research documents, whether they are published or not. The documents may come from teaching and research institutions in France or abroad, or from public or private research centers.
L'archive ouverte pluridisciplinaire HAL, est destinée au dépôt et à la diffusion de documents scientifiques de niveau recherche, publiés ou non, émanant des établissements d'enseignement et de recherche français ou étrangers, des laboratoires publics ou privés. 


\title{
Optical index of fault tolerant routings in WDM networks
}

\author{
S. Bessy \\ Laboratoire LIRMM - Université Montpellier 2, \\ 161, rue Ada, 34000 Montpellier, \\ France \\ bessy@lirmm.fr \\ and \\ C. Lepelletier, \\ Projet Mascotte, CNRS/INRIA/UNSA, \\ INRIA Sophia-Antipolis, \\ 2004 route des Lucioles BP 93, \\ 06902 Sophia-Antipolis Cedex, \\ France
}

June 9, 2009

\begin{abstract}
Maňuch and Stacho [7] introduced the problem of designing $f$-tolerant routings in optical networks, i.e., routings which still satisfy the given requests even if $f$ failures occur in the network. In this paper, we provide $f$-tolerant routings in complete and complete balanced bipartite optical networks, optimal according to two parameters: the arc-forwarding index and the optical index. These constructions use tools from design theory and graph theory and improve previous results of Dinitz, Ling and Stinson [4] for the complete network, and Gupta, Maňuch and Stacho [5] for the complete balanced bipartite network.
\end{abstract}

Keywords: optical networks, forwarding and optical indices, routing, fault tolerance 


\section{Introduction}

In this paper, we are interested in a problem arising in the design of optical networks. Using models of graph theory and design theory, this topic has been of considerable interest over the last decade (see [1], [2] or [5] for instance). Readers may refer to [1] for a background review of optical networks. The model studied in this article is valid for the so-called wavelength division multiplexing (or WDM) optical network. Such a network is modeled by a symmetric directed graph with arcs representing the fiber-optic links. A request in the network is an ordered pair of graph nodes, representing a possible communication in the network. A set of different requests is an instance in the network. For each request of the instance, we have to select a routing directed path to satisfy it, and the set of all selected paths forms a routing set according to the instance. To make the communications possible, a wavelength is allocated to each routing path, such that two paths sharing an arc do not carry the same wavelength; otherwise the corresponding communications could be perturbed. Given a routing set related to the wavelength assignment, we can define two classical invariants. The arc-forwarding index of the routing set is the maximum number of paths sharing the same arc. In the network, there is a general bound on the number of wavelengths which can transit at the same time in a fiber-optic link, corresponding to the admissible maximal arc-forwarding index. The other invariant, called the optical index of the routing set, is the minimum number of wavelengths to assign to the routing paths in order to ensure that there is no interference in the network. The main challenge here is to provide, for a given instance, a routing set which minimizes the arc-forwarding index or the optical index, or both if possible.

Our work is a contribution to a variant of this problem, introduced by Maňuch and Stacho [7], in which we focus on possible breakdowns of nodes in the network. Precisely, for a given fixed integer $f$, we have to provide, for every request, not just one directed path to satisfy it, but rather a set of $f+1$ directed paths with the same beginning and end nodes (corresponding to the request) and which are internally disjoint. In this routing, if $f$ nodes break down, every request between the remaining nodes could still be satisfied by a previously selected routing path which contains no failed component. Such a routing set of directed paths is called an $f$-fault tolerant routing or an $f$-tolerant routing.

In this paper, we focus on the very special cases of complete symmetric directed graphs and 
complete balanced bipartite symmetric directed graphs. Moreover, we only study the case of all-to-all communication, i.e., where the instance of the problem is the set of all ordered pairs of nodes of the network. Some results on these problems were presented by Gupta, Mañuch and Stacho [5] and Dinitz, Ling and Stinson [4]. We improve these results: for complete symmetric directed graphs, we show that optimal routings for the arc-forwarding index given in [5] are also optimal for the optical index. And for complete balanced bipartite symmetric directed graphs, we provide routings that are optimal for both parameters.

\section{Preliminaries}

In this section, we specify the previous definitions and formalize the problem. For the purpose of the paper, we only describe the case of all-to-all communication, but the notions can be extended to any kind of instances. We mainly use the notations proposed in [5].

We model an all-optical network as a symmetric directed graph $D=(V(D), A(D))$, where $V(D)$ is the vertex set of $D$ and $A(D)$ is the arc set with the additional property that if $(u, v) \in A(D)$ then $(v, u) \in A(D)$. If no confusion is possible, we simply write $V$ and $A$ instead of $V(D)$ and $A(D)$, respectively. All paths and circuits are considered as oriented.

A directed graph $D$ is strongly connected if, for every two vertices $x$ and $y$ of $D$, there is a path from $x$ to $y$ in $D$. In a symmetric directed graph, strong connectivity is equivalent to connectivity of the underlying non-oriented graph. So, for an integer $k \geq 1$, a symmetric directed graph $D$ is $k$-connected if, for every set $\left\{x_{1}, \ldots, x_{k-1}\right\}$ of vertices of $D, D \backslash\left\{x_{1}, \ldots, x_{k-1}\right\}$ is strongly connected.

For a fixed positive integer $f$, an $f$-tolerant routing in $D$ is a set of paths:

$$
\mathcal{R}=\left\{P_{i}(u, v): u, v \in V, u \neq v, i=0, \ldots, f\right\}
$$

where, for each pair of distinct vertices $u, v \in V$, the paths $P_{0}(u, v), \ldots, P_{f}(u, v)$ are internally vertex disjoint. Note that such a set of paths exists if and only if the connectivity of the directed graph is large enough (at least $f+1$ ), which will be the case in complete and complete bipartite networks for suitable $f$.

The basic parameters for WDM optical networks, the arc-forwarding index and the optical index, are generalized in $f$-tolerant routings. The load of an arc in $\mathcal{R}$ is the number of directed 
paths of $\mathcal{R}$ containing it. By extension, the maximum load over all the $\operatorname{arcs}$ of $D$ is the load of the routing, which is also called the arc-forwarding index of $\mathcal{R}$ and is denoted by $\pi(\mathcal{R})$. Each path of $\mathcal{R}$ receives a wavelength in the network to enable the communication and, to avoid interference, two paths sharing an arc do not receive the same wavelength. Like graph coloring, we speak about wavelengths as colors to assign to the paths of $\mathcal{R}$. Finally, the optical index of $\mathcal{R}$, denoted $w(\mathcal{R})$, is the minimum number of wavelengths to assign to paths of $\mathcal{R}$ so that no two paths sharing an arc receive the same wavelength. In other words, $w(\mathcal{R})$ is exactly the chromatic number of the graph with vertex set $\mathcal{R}$ and where two paths of $\mathcal{R}$ are linked if they share the same arc of $D$ (known as the path graph of $\mathcal{R}$ ).

The goal is to minimize $\pi(\mathcal{R})$ and $w(\mathcal{R})$. So the $f$-tolerant arc-forwarding index of $D$ and the $f$-tolerant optical index of $D$ are respectively defined by:

$$
\begin{aligned}
& \pi_{f}(D)=\min _{\mathcal{R}} \pi(\mathcal{R}) \\
& w_{f}(D)=\min _{\mathcal{R}} w(\mathcal{R})
\end{aligned}
$$

where the minima span all the possible routing sets $\mathcal{R}$. A routing set achieving one of the bounds is said to be optimal for the arc-forwarding index or optimal for the optical index, respectively.

For a routing set $\mathcal{R}$, all paths sharing the same arc must receive different wavelengths in the computation of $w(\mathcal{R})$. In particular, we have $\pi(\mathcal{R}) \leq w(\mathcal{R})$. By considering a routing set which is optimal for the optical index, we obtain $\pi_{f}(D) \leq w_{f}(D)$. The equality was conjectured by Maňuch and Stacho [7].

Conjecture 1 (J. Man̆uch, L. Stacho, 2003, [7]) Let $D$ be a symmetric directed $k$-connected graph. For any $f, 0 \leq f<k$, we have $\pi_{f}(D)=w_{f}(D)$.

For $f=0$ (without tolerating any faults), the conjecture was previously raised by Beauquier et al. [1].

Let $K_{n}^{\star}$ denote the complete symmetric directed graph with vertex set $\left\{x_{1}, \ldots, x_{n}\right\}$ and arc set $\left\{x_{i} x_{j}: i \neq j\right\}$. The complete balanced bipartite symmetric digraph $K_{n, n}^{\star}$ is the directed graph on vertex set $X \cup Y$ with $X=\left\{x_{1}, \ldots, x_{n}\right\}$ and $Y=\left\{y_{1}, \ldots, y_{n}\right\}$ and $\operatorname{arc} \operatorname{set}\{x y, y x: x \in X, y \in Y\}$. The arc-forwarding indices of $K_{n}^{\star}$ and $K_{n, n}^{\star}$ were computed by Gupta, Maňuch and Stacho in [5]. 
Moreover, they give $w_{f}\left(K_{n}^{\star}\right)$ and $w_{f}\left(K_{n, n}^{\star}\right)$ up to a multiplicative factor. In [4], Dinitz, Ling and Stinson compute, among other things, $w_{f}\left(K_{n}^{\star}\right)$ up to an additive constant in some cases.

In the next two sections, we provide exact values for $w_{f}\left(K_{n}^{\star}\right)$ and $w_{f}\left(K_{n, n}^{\star}\right)$ and hence prove Conjecture 1 for the complete symmetric directed graph and the complete balanced bipartite symmetric directed graph.

\section{Complete optical network}

It is easy to provide a lower bound for the arc-forwarding index of $K_{n}^{\star}$. Indeed, any two vertices $x$ and $y$ of $K_{n}^{\star}$ have to be linked in an $f$-tolerant routing by $f+1$ internally disjoint paths. If one of these paths has length one (the direct arc $x y$ ), all the others have length at least two, and at least $2 f+1$ arcs are needed to ensure $f$-tolerant communication from $x$ to $y$. So, by an average argument, one arc of $K_{n}^{\star}$ must have load at least $2 f+1$, providing $w_{f}\left(K_{n}^{\star}\right) \geq 2 f+1$. In the case of $K_{n}^{\star}$, Gupta, Mañuch and Stacho prove that this lower bound gives exactly the value of the arc-forwarding index. Indeed, they construct $f$-tolerant routings through families of independent idempotent Latin squares in [5], which are optimal for the arc-forwarding index.

Theorem 2 (A. Gupta, J. Maňuch, L.Stacho, 2005, [5]) For every $f$ with $0 \leq f \leq n-2$, we have $\pi_{f}\left(K_{n}^{\star}\right)=2 f+1$.

They also partially bound the optical index of their $f$-tolerant routings, proving that $w_{f}\left(K_{n}^{\star}\right) \leq$ $3 f+1$ for some $f$. This result was improved by Dinitz, Ling and Stinson [4], who gave a better multiplicative factor for some infinite sets of values of $n$ and the optimal index up to an additive constant for another infinite set of values of $n$. We improve these results by showing that every $f$-tolerant routing set of $K_{n}^{\star}$ which is optimal for the arc-forwarding index is also optimal for the optical index.

Theorem 3 For every $f, 0 \leq f \leq n-2$, and every $f$-tolerant routing set $\mathcal{R}$ of $K_{n}^{\star}$ with $\pi(\mathcal{R})=$ $\pi_{f}\left(K_{n}^{\star}\right)=2 f+1$, we have $w(\mathcal{R})=2 f+1$. In particular, we have $w_{f}\left(K_{n}^{\star}\right)=\pi_{f}\left(K_{n}^{\star}\right)=2 f+1$.

Proof. Let $f$ be fixed with $0 \leq f \leq n-2$ and consider an $f$-tolerant routing $\mathcal{R}$ of $K_{n}^{\star}$ which is optimal for the arc-forwarding index, i.e., of value $2 f+1$. By the tightness of the lower bound, for any two vertices $x$ and $y$ of $K_{n}^{\star}$ there is exactly one path $x y$ of length 1 and $f$ paths of length 
2 from $x$ to $y$ in $\mathcal{R}$ (otherwise, summing up the total load gives $\pi(\mathcal{R}) \geq 2 f+2$ ). Hence, every arc of $K_{n}^{\star}$ has a load of exactly $2 f+1$ and appears in one path of length 1 and $2 f$ paths of length two in $\mathcal{R}$. Now, define the graph $H$ with vertex set being the set of the $\operatorname{arcs}$ of $K_{n}^{\star}$ and link two $\operatorname{arcs}$ of $K_{n}^{\star}$ if they belong to the same path of $\mathcal{R}$ of length 2 . Thus, we have a one-to-one correspondence between the edge set of $H$ and the paths of length 2 of $\mathcal{R}$. Since each arc of $K_{n}^{\star}$ belongs to exactly $2 f$ paths of $\mathcal{R}$ of length $2, H$ is regular with degree $2 f$. By Vizing's Theorem (see [3] or [9]), the edges of $H$ can be colored with $2 f+1$ distinct colors such that any two adjacent edges receive distinct colors. This provides a coloring of the paths of length 2 of $\mathcal{R}$ with $2 f+1$ colors. To conclude, a path of length 1 of $\mathcal{R}$ intersects exactly $2 f$ paths of length 2 and we can color this path with the remaining color.

Moreover, the edge-coloring provided by Vizing's Theorem can be computed in polynomial time (polynomial in the size of the input graph, $H$ here). So, given an optimal routing for the arc-forwarding index, this proof gives a polynomial algorithm (polynomial in $n$ and $f$ ) to obtain a wavelength assignment for a routing which is optimal for the optical index.

\section{Complete balanced bipartite optical network}

In this section, we compute the exact optical index of $K_{n, n}^{\star}$ and thus prove Conjecture 1 for this family of graphs. This improves the result in [5], where the upper bound given on the optical index of $K_{n, n}^{\star}$ is $20 \%$ higher than the conjectured optimal value.

Theorem 4 For any $n \geq 1$ and any $f$ with $0 \leq f \leq n-1$, we have $w_{f}\left(K_{n, n}^{\star}\right)=\pi_{f}\left(K_{n, n}^{\star}\right)$.

To prove Theorem 4, we provide a routing set for $K_{n, n}^{\star}$ which is optimal both for the arcforwarding index and the optical index. The construction depends on the values of $n$ and $f$. Recall that $X \cup Y$ denotes the canonical partition of $K_{n, n}^{\star}$, with $X=\left\{x_{1}, \ldots, x_{n}\right\}$ and $Y=$ $\left\{y_{1}, \ldots, y_{n}\right\}$. For convenience, indices of the vertices of $X$ and $Y$ are computed modulo $n$.

\subsection{Routing set and arc-forwarding index}

We use the paths of minimum length to route in $K_{n, n}^{\star}$. Indeed, for two vertices $x$ and $y$, we use paths of length 2 when $x$ and $y$ belong to the same partite set of $K_{n, n}^{\star}$ and one path of length 1 
and other paths of length 3 (if $f>0$ ) when $x$ and $y$ do not belong to the same partite set. The main challenge here is to pack the paths of length 3 in order to provide the right optical index. This will be achieved by using the following decomposition result.

Theorem 5 (Tillson, 1980, [8]) The arcs of $K_{n}^{\star}$ can be partitioned into Hamiltonian circuits if and only if $n \neq 4$ and $n \neq 6$.

For $n \neq 4$ and $n \neq 6,\left\{C_{1}, \ldots, C_{n-1}\right\}$ denotes a set of $n-1$ Hamiltonian circuits which partition the $\operatorname{arcs}$ of $K_{n}^{\star}$. Let $\{1, \ldots, n\}$ denote the vertex set of $K_{n}^{\star}$. Moreover, for a vertex $i$ of $K_{n}^{\star}$ and a circuit $C_{k}$, the out-neighbor of $i$ in $C_{k}$ is denoted $C_{k}(i)$. We use $C_{k}$ as a functional notation: for $p \geq 1, C_{k}^{p}(i)=C_{k}\left(C_{k}^{p-1}(i)\right)$. Moreover, we compute the powers of $C_{k}$ modulo $n$, in particular $C_{k}^{0}(i)=i$ and $C_{k}^{-1}(i)$ is the in-neighbor of $i$ in $C_{k}$.

The previous Hamiltonian decomposition is used to route paths of length 3 in $K_{n, n}^{\star}$. For paths of length 2 , we use a Latin square $A$ of order $n$, i.e., a $n \times n$ matrix in which each row and each column is a permutation of the set $\{1, \ldots, n\}$. Moreover, we require $A$ to be idempotent: for every $i, 1 \leq i \leq n$, we have $A(i, i)=i$. An idempotent Latin square exists for each value of $n$, except for $n=2$ (see [6], Chapter 2, for an explicit construction). For $0 \leq k \leq n-1$, $M_{k}=\left\{x_{i} y_{i+k}, y_{i+k} x_{i}: 1 \leq i \leq n\right\}$, denotes the symmetric orientations of $n$ disjoint matchings which partition the arcs of $K_{n, n}^{\star}$ (see Figure 1). The indices of $M_{k}$ are computed modulo $n$. In our figures, we represent two symmetric arcs by a (non-oriented) edge.

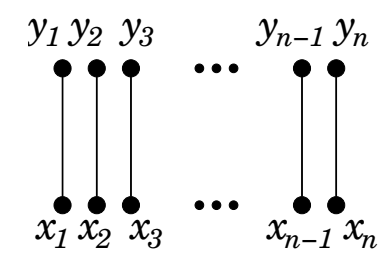

$M_{0}$

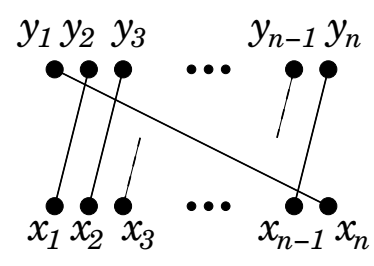

$M_{1}$

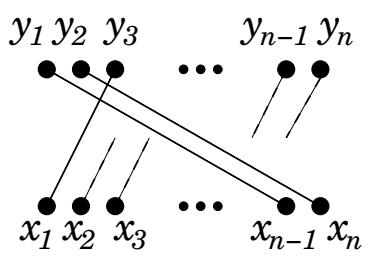

$M_{2}$

Figure 1: Some matchings $M_{k}$.

Now, for $n \neq 2, n \neq 4$ and $n \neq 6$, we describe three kinds of paths to construct the routing set: 
- Paths of length 1 between vertices which belong to different partite sets of $K_{n, n}^{\star}$ :

$$
\begin{gathered}
\mathcal{D}_{0}[X, Y]=\left\{x_{i} y_{j}: 1 \leq i \leq n, 1 \leq j \leq n\right\} \\
\mathcal{D}_{0}[Y, X]=\left\{y_{i} x_{j}: 1 \leq i \leq n, 1 \leq j \leq n\right\} \\
\mathcal{D}_{0}=\mathcal{D}_{0}[X, Y] \cup \mathcal{D}_{0}[Y, X]
\end{gathered}
$$

- Paths of length 3 between vertices which belong to different partite sets of $K_{n, n}^{\star}$, for $1 \leq k \leq$ $n-1$ :

$$
\begin{gathered}
\mathcal{D}_{k}[X, Y]=\left\{x_{i} y_{C_{k}^{-1}(j)} x_{C_{k}(i)} y_{j}: 1 \leq i \leq n, 1 \leq j \leq n\right\} \\
\mathcal{D}_{k}[Y, X]=\left\{y_{i} x_{C_{k}^{-1}(j)} y_{C_{k}(i)} x_{j}: 1 \leq i \leq n, 1 \leq j \leq n\right\} \\
\mathcal{D}_{k}=\mathcal{D}_{k}[X, Y] \cup \mathcal{D}_{k}[Y, X]
\end{gathered}
$$

- Paths of length 2 between vertices which belong to the same partite set of $K_{n, n}^{\star}$, for $0 \leq k \leq$ $n-1$ :

$$
\begin{gathered}
\mathcal{S}_{k}[X, X]=\left\{x_{i} y_{A(i, j)+k} x_{j}: 1 \leq i \leq n, 1 \leq j \leq n, i \neq j\right\} \\
\mathcal{S}_{k}[Y, Y]=\left\{y_{i} x_{A(i, j)+k+\lceil n / 2\rceil} y_{j}: 1 \leq i \leq n, 1 \leq j \leq n, i \neq j\right\} \\
\mathcal{S}_{k}=\mathcal{S}_{k}[X, X] \cup \mathcal{S}_{k}[Y, Y]
\end{gathered}
$$

And for a fixed $f, 0 \leq f \leq n-1$, we define the routing set $\mathcal{R}_{f}$ by

$$
\mathcal{R}_{f}=\bigcup_{k=0}^{f}\left(\mathcal{S}_{k} \cup \mathcal{D}_{k}\right)
$$

Note that, by construction, for distinct vertices $x$ and $y$ of $K_{n, n}^{\star}, \mathcal{R}_{f}$ contains exactly $f+1$ internally disjoint paths from $x$ to $y$. So, $\mathcal{R}_{f}$ is an $f$-tolerant routing for an all-to-all instance in $K_{n, n}^{\star}$. Moreover, note that every arc of $K_{n, n}^{\star}$ appears exactly once in $\mathcal{D}_{0}$ and three times in $\mathcal{D}_{k}$ for $1 \leq k \leq n-1$ (for instance the arc $x_{i} y_{j}$ appears in $\mathcal{D}_{k}$ in paths from $x_{i}$ to $y_{C_{k}(j)}$, from $x_{C_{k}^{-1}(i)}$ to $y_{j}$ and from $y_{C_{k}^{-1}(j)}$ to $\left.x_{C_{k}(i)}\right)$. For $0 \leq k \leq n$, the routing $\mathcal{S}_{k}$ behaves slightly differently: $\mathcal{S}_{k}[X, X]$ contains only pairwise arc-disjoint paths and the same holds for $\mathcal{S}_{k}[Y, Y]$. Moreover, $\mathcal{S}_{k}[X, X]$ and $M_{k}$ are disjoint and every arc not in $M_{k}$ appears exactly once in $\mathcal{S}_{k}[X, X]$; on the other hand, $\mathcal{S}_{k}[Y, Y]$ and $M_{k+\lceil n / 2\rceil}$ are disjoint and every arc not in $M_{k+\lceil n / 2\rceil}$ appears exactly once in $\mathcal{S}_{k}[X, X]$. Using these remarks, we can give the arc-forwarding index of $\mathcal{R}_{f}$. The computation of $\pi_{f}\left(K_{n, n}^{\star}\right)$ was obtained in [5]. 
Lemma 6 (A. Gupta, J. Mañuch, L. Stacho, 06, [5]) The arc-forwarding of $K_{n, n}^{\star}$ is:

$$
\pi_{f}\left(K_{n, n}^{\star}\right)= \begin{cases}5 f+3 & \text { for } 0 \leq f \leq\lceil n / 2\rceil-2 \\ 5 f+2 & \text { for }\lceil n / 2\rceil-1 \leq f \leq n-2 \\ 5 f+1 & \text { for } f=n-1\end{cases}
$$

In fact, we prove that $\mathcal{R}_{f}$ is optimal for the arc-forwarding index.

Lemma 7 For every $f, 0 \leq f \leq n-1$, the routing set $\mathcal{R}_{f}$ satisfies $\pi\left(\mathcal{R}_{f}\right)=\pi_{f}\left(K_{n, n}^{\star}\right)$.

Proof. For $0 \leq f \leq\lceil n / 2\rceil-2$, every arc of $K_{n, n}^{\star}$ appears at least 5 times in $\mathcal{S}_{k} \cup \mathcal{D}_{k}$ for $1 \leq k \leq f$ and 3 times in $\mathcal{S}_{0} \cup \mathcal{D}_{0}$, so the computation of $\pi\left(\mathcal{R}_{f}\right)$ is clear. For $f \geq\lceil n / 2\rceil-1$, every arc of $K_{n, n}^{\star}$ is in a matching $M_{k}$ or $M_{k+\lceil n / 2\rceil}$ for some $k, 0 \leq k \leq f$ and thus is not in a path of one of the $\mathcal{S}_{k}[X, X]$ or $\mathcal{S}_{k}[Y, Y]$. We then save 1 in the computation of $\pi\left(\mathcal{R}_{f}\right)$ in these cases. Finally, if $f=n-1$, every arc of $K_{n, n}^{\star}$ is in a matching $M_{k}$ and in a matching $M_{k^{\prime}+\lfloor n / 2\rfloor}$ for some suitable $k$ and $k^{\prime}$ in $\{0, \ldots, n-1\}$. So, every $\operatorname{arc}$ of $K_{n, n}^{\star}$ is not in any path of $\mathcal{S}_{k}[X, X]$ and not in any path of $\mathcal{S}_{k^{\prime}}[Y, Y]$, and we save two in the computation of $\pi\left(K_{n, n}^{\star}\right)$.

We then have a lower bound for the optical index of $K_{n, n}^{\star}$, and now we prove that the routing set $\mathcal{R}_{f}$ achieves this bound.

\subsection{Packing the paths of $\mathcal{R}_{f}$}

A color class of paths of $\mathcal{R}_{f}$ is set of paths which are pairwise arc-disjoint. To construct the different color classes, we need the following notations and definitions. To indicate a path or a set of paths of $\mathcal{R}_{f}$, we always specify the subset $\mathcal{D}_{k}$ or $\mathcal{S}_{k}$ it belongs to. For instance, $\mathcal{S}_{0}[X, X]\left\{x_{1} y_{A(1,2)} x_{2}\right\}$ is the path $x_{1} y_{A(1,2)} x_{2}$ of $\mathcal{S}_{0}[X, X]$ and $\mathcal{D}_{0}\left\{M_{1}\right\}$ is the set of paths in $M_{1}$ of $\mathcal{D}_{0}$. We use the notation $\star$ as a 'joker' instead of all the possible path names. For instance, $\mathcal{S}_{0}[X, X]\left\{\star y_{1} \star\right\}$ stands for all paths of $\mathcal{S}_{0}[X, X]$ whose intermediate vertex is $y_{1}$. We specially focus on two particular subsets of paths. For $k \geq 1$ and $x_{i}$, a vertex of $K_{n, n}^{\star}, \mathcal{D}_{k}[X, Y]\left\{x_{i} \star x_{C_{k}(i)} \star\right\}$ contains the $n$ paths of $\mathcal{D}_{k}$ which start at $x_{i}$ and whose third vertex is $x_{C_{k}(i)}$. As $\left(C_{k}(1), C_{k}(2), \ldots, C_{k}(n)\right)$ is a permutation of $(1,2, \ldots, n)$, note that these paths are pairwise arc-disjoint and that they cover exactly all arcs beginning at $x_{i}$, all arcs beginning at $x_{C_{k}(i)}$ and all arcs ending at $x_{C_{k}(i)}$. Moreover, we have 
$\bigcup_{l=0}^{n-1} \mathcal{D}_{k}[X, Y]\left\{x_{C_{k}^{l}(1)} \star x_{C_{k}^{l+1}(1)} \star\right\}=\mathcal{D}_{k}[X, Y]$. The set $\mathcal{D}_{k}[X, Y]\left\{x_{i} \star x_{C_{k}(i)} \star\right\}$ and its representation on $X$, which shows the saturated in and out-neighborhood, are depicted in Figure 2. Similarly, we will use the sets $\mathcal{D}_{k}[X, Y]\left\{\star y_{i} \star y_{C_{k}(i)}\right\}, \mathcal{D}_{k}[Y, X]\left\{\star x_{i} \star x_{C_{k}(i)}\right\}$ and $\mathcal{D}_{k}[Y, X]\left\{y_{i} \star y_{C_{k}(i)} \star\right\}$.

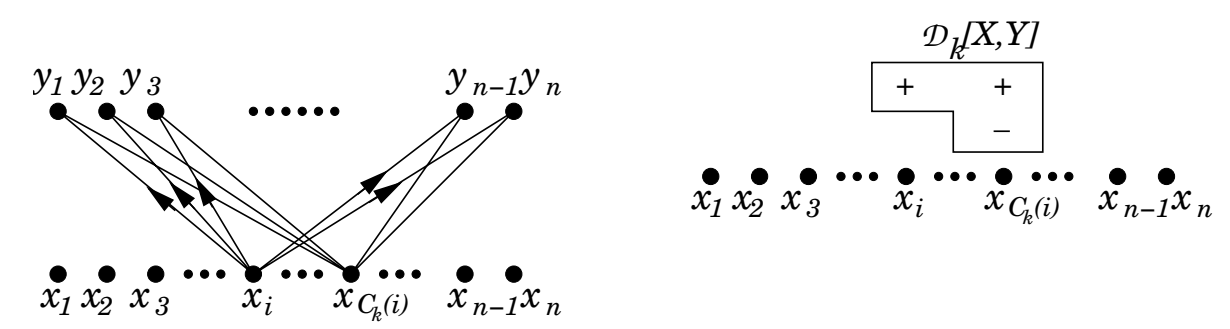

Figure 2: The set of paths $\mathcal{D}_{k}[X, Y]\left\{x_{i} \star x_{C_{k}(i)} \star\right\}$ and its representation on $X$.

In addition, for $0 \leq k \leq n-1$ and $1 \leq i \leq n, \overline{\mathcal{S}_{k}[Y, Y]}\left\{\star x_{i} \star\right\}$ denotes the set of paths $\mathcal{S}_{k}[Y, Y]\left\{\star x_{i} \star\right\} \cup \mathcal{D}_{0}\left\{x_{i} y_{i+k+\lceil n / 2\rceil}, y_{i+k+\lceil n / 2\rceil} x_{i}\right\}$. As $\mathcal{S}_{k}[Y, Y]$ and $M_{k+\lceil n / 2\rceil}$ are disjoint, $\overline{\mathcal{S}_{k}[Y, Y]}\left\{\star x_{i} \star\right\}$ contains only arc-disjoint paths. Moreover, it contains exactly all arcs beginning and ending at $x_{i}$, and we have $\bigcup_{i=1}^{n} \overline{\mathcal{S}_{k}[Y, Y]}\left\{\star x_{i} \star\right\}=\mathcal{S}_{k}[Y, Y] \cup \mathcal{D}_{0}\left\{M_{k+\lceil n / 2\rceil}\right\}$. This set and the representation of $\overline{\mathcal{S}_{k}[Y, Y]}\left\{\star x_{i} \star\right\}$ on $X$ are shown in Figure 3. Similarly, we will use the sets $\overline{\mathcal{S}_{k}[X, X]}\left\{\star y_{i} \star\right\}$, disjoint from $\mathcal{D}_{0}\left\{M_{k}\right\}$.
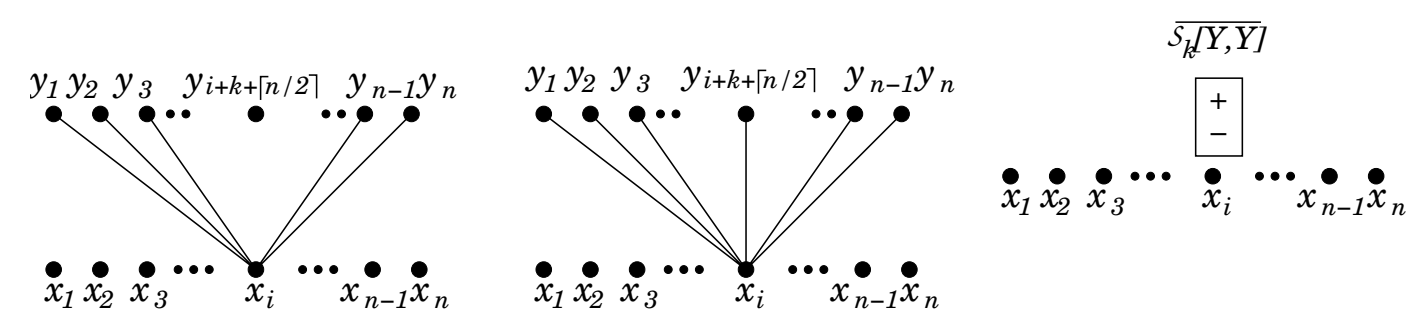

Figure 3: The sets $\mathcal{S}_{k}[Y, Y]\left\{\star x_{i} \star\right\}, \overline{\mathcal{S}_{k}[Y, Y]}\left\{\star x_{i} \star\right\}$ and the representation of $\overline{\mathcal{S}_{k}[Y, Y]}\left\{\star x_{i} \star\right\}$ on $X$.

Now we can define the colors classes. They are constructed differently according to the value of $f$ : we distinguish three main cases following the residue of $n$ modulo 3 . However, some particular cases occur: $n=2$ due to the non-existence of an idempotent Latin square of order 
2, $n=4$ and $n=6$ which are exceptions to Tillson's Theorem, and $n=1$ and $n=5$ for which the techniques used in general cases $n \equiv 1(\bmod 3)$ and $n \equiv 2(\bmod 3)$ cannot be applied. Moreover, for any value of $n$, the case $f=0$ is different from the other cases.

\subsection{Routings without fault}

The case $f=0$ is simpler than other cases in the sense that the paths of length 1 form a proper color class, whereas for $f>0$ these paths are used to complete other color classes. More precisely, $\mathcal{R}_{0}=\mathcal{D}_{0} \cup \mathcal{S}_{0}$ and to obtain the optical index, we assign one different color to each of these sets of paths: $\mathcal{D}_{0}, \mathcal{S}_{0}[X, X]$ and $\mathcal{S}_{0}[Y, Y]$. So, for every $n \geq 1$, we have $w_{0}\left(K_{n, n}^{\star}\right)=\pi_{0}\left(K_{n, n}^{\star}\right)=3$.

\subsection{Optical index in case $n \equiv 0(\bmod 3)$}

This is the simplest case, so paths of length 2 and 3 can be packed separately. For any $k$, $1 \leq k \leq n-1$, to color paths of length 3 , we define:

$$
\begin{aligned}
c_{k}^{3} & =\bigcup_{t=0}^{n / 3-1}\left(\mathcal{D}_{k}[Y, X]\left\{\star x_{C_{k}^{3 t}(1)} \star x_{C_{k}^{3 t+1}(1)}\right\} \cup \mathcal{D}_{k}[X, Y]\left\{x_{C_{k}^{3 t+1}(1)} \star x_{C_{k}^{3 t+2}(1)} \star\right\}\right) \\
c_{k}^{4} & =\bigcup_{t=0}^{n / 3-1}\left(\mathcal{D}_{k}[Y, X]\left\{\star x_{C_{k}^{3 t+1}(1)} \star x_{C_{k}^{3 t+2}(1)}\right\} \cup \mathcal{D}_{k}[X, Y]\left\{x_{C_{k}^{3 t+2}(1)} \star x_{C_{k}^{3 t+3}(1)} \star\right\}\right) \\
c_{k}^{5} & =\bigcup_{t=0}^{n / 3-1}\left(\mathcal{D}_{k}[Y, X]\left\{\star x_{C_{k}^{3 t+2}(1)} \star x_{C_{k}^{3 t+3}(1)}\right\} \cup \mathcal{D}_{k}[X, Y]\left\{x_{C_{k}^{3 t+3}(1)} \star x_{C_{k}^{3 t+4}(1)} \star\right\}\right)
\end{aligned}
$$

In Figure 4, we give the representation on $X$ of $c_{k}^{3}$, where the vertices of $X$ are sorted along $C_{k}$. A shift of one vertex on the right side (modulo $n$ ) of the sets of paths gives the representation of $c_{k}^{4}$, for the same $k$. And a shift of two vertices gives the representation of $c_{k}^{5}$. Then, note that every path of $\mathcal{D}_{k}[X, X]$ belongs to one of the class $c_{k}^{3}, c_{k}^{4}$ or $c_{k}^{5}$. For $k \in\{0, \ldots, n-1\}$, we also define $c_{k}^{1}=\overline{\mathcal{S}_{k}[Y, Y]}$. Now, we pack the remaining paths of length 1 and 2 in classes $c_{k}^{2}$ according to the value of $f$.

- If $1 \leq f \leq\lceil n / 2\rceil-2$, for $0 \leq k \leq f$, we fix:

$$
c_{k}^{2}=\mathcal{S}_{k}[X, X]
$$




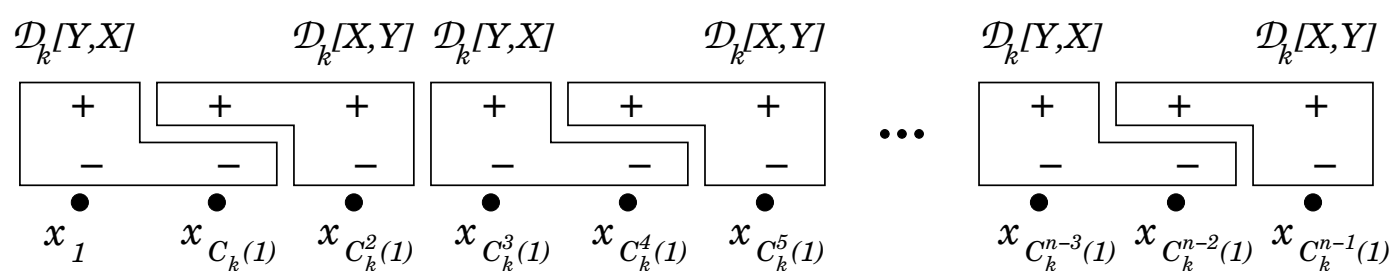

Figure 4: The representation on $X$ of the color class $c_{k}^{3}$, where the vertices of $X$ are sorted along $C_{k}$.

All paths of $\mathcal{D}_{0}$ have not been used in the sets $c_{0}^{1} \cup c_{0}^{2} \cup \bigcup_{k=1}^{f}\left\{c_{k}^{1}, c_{k}^{2}, c_{k}^{3}, c_{k}^{4}, c_{k}^{5}\right\}$ (sole $\mathcal{D}_{0}\left\{M_{\lceil n / 2\rceil}\right\}, \mathcal{D}_{0}\left\{M_{1+\lceil n / 2\rceil}\right\}, \ldots, \mathcal{D}_{0}\left\{M_{f+\lceil n / 2\rceil}\right\}$ are used). So, we put the remaining paths of $\mathcal{D}_{0}$ in a class $c_{0}^{3}$, and we obtain, in this case, $w_{f}\left(K_{n, n}^{\star}\right)=5 f+3$.

- If $\lceil n / 2\rceil-1 \leq f \leq n-2$, we have enough space to pack the paths of length one in the other classes. Indeed, $c_{0}^{1} \cup c_{0}^{2} \cup \bigcup_{k=1}^{f}\left\{c_{k}^{1}, c_{k}^{3}, c_{k}^{4}, c_{k}^{5}\right\}$ does not contain the direct paths $\mathcal{D}_{0}\left\{M_{f+1+\lceil n / 2\rceil}\right\}, \mathcal{D}_{0}\left\{M_{f+2+\lceil n / 2\rceil}\right\}, \ldots, \mathcal{D}_{0}\left\{M_{\lceil n / 2\rceil-1}\right\}$, with $n \leq f+1+\lceil n / 2\rceil \leq n+\lceil n / 2\rceil-$ 1 (note that indices of $M_{i}$ are computed modulo $n$ ). So, for $0 \leq k \leq f$, we set:

$$
\begin{array}{ll}
c_{k}^{2}=\overline{\mathcal{S}_{k}[X, X]} & \text { if } f+1+\lceil n / 2\rceil-n \leq k \leq\lceil n / 2\rceil-1 \\
c_{k}^{2}=\mathcal{S}_{k}[X, X] & \text { else }
\end{array}
$$

So, all paths of $\mathcal{D}_{0}$ are used, and we obtain $w_{f}\left(K_{n, n}^{\star}\right)=5 f+2$.

- If $f=n-1$, we should save another color. This time, all paths of length 1 are packed in $\bigcup_{k=0}^{n-1} c_{k}^{2}$ and the second saved color is obtained by optimally packing the set of paths $\left\{\mathcal{S}_{k}[X, X]: 0 \leq k \leq n-1\right\}$ using only $n-1$ colors. First, we complete the color class $c_{1}^{2}$. We start by covering all the arcs beginning at $x_{1}$ and all the arcs ending at $x_{2}$, which can be done by placing in $c_{1}^{2}$ all the paths from $x_{1}$ to $x_{2}: \bigcup_{i=0}^{n-1} \mathcal{S}_{i}[X, X]\left\{x_{1} y_{A(1,2)+i} x_{2}\right\}$ (corresponding to the paths of $\bigcup_{i=0}^{n-1} \mathcal{S}_{i}[X, X]$ which use the value $A(1,2)$ in the matrix $A$ ). Then, we focus on the arcs beginning at $x_{2}$ and the arcs ending at $x_{3}$ using the paths: $\bigcup_{i=0}^{n-1} \mathcal{S}_{i}[X, X]\left\{x_{2} y_{A(2,3)+i} x_{3}\right\}$ (paths using $A(2,3)$ ). Subsequently, we cover the arcs beginning at $x_{p}$ and the arcs ending at $x_{p+1}$ for $p=1,2, \ldots, n$ (corresponding to all paths using values $A(p, p+1)$ in $A)$. So, $c_{1}^{2}$ forms a color class which covers exactly once all $\operatorname{arcs}$ of $K_{n, n}^{\star}$. Once $c_{1}^{2}$ is complete, we proceed in the same way to obtain $c_{2}^{2}$, using, this time, for $p=1,2, \ldots, n$, the paths of $\bigcup_{i=0}^{n-1} \mathcal{S}_{i}[X, X]$ obtained through the values $A(p, p+2)$ 
to cover the arcs from $x_{p}$ and those to $x_{p+2}$.

In general way, $1 \leq k \leq n-1, c_{k}^{2}$ is constructed using the paths obtained through the values $A(p, p+k)$, for $p=1,2, \ldots, n$. More precisely, we define:

$$
c_{k}^{2}=\bigcup_{j=1}^{n} \bigcup_{i=0}^{n-1} \mathcal{S}_{i}[X, X]\left\{x_{j} \star x_{j+k}\right\}
$$

Finally, we obtain the color classes $\left\{c_{0}^{1}\right\} \cup \bigcup_{k=1}^{n-1}\left\{c_{k}^{1}, c_{k}^{2}, c_{k}^{3}, c_{k}^{4}, c_{k}^{5}\right\}$ which give the optimal value for the optical index: $w_{f}\left(K_{n, n}^{\star}\right)=5 f+1$.

\subsection{Optical index in case $n \equiv 1(\bmod 3)$}

This time a color class cannot be composed with only paths of length 3 . For $1 \leq k \leq n-1$, we pack together a maximum number of paths of length 3 (i.e., from $\mathcal{D}_{k}$ ) in classes $c_{k}^{3}, c_{k}^{4}$ and $c_{k}^{5}$, which we supplement with paths of length 2 from $c_{k}^{1}$ (i.e., from $\mathcal{S}_{k}$ ). Consequently, $c_{k}^{1}$ contains the main part of paths of length 2 and the remaining paths of length 3 , which is possible as soon as $n \geq 3$. Precisely, for $n \geq 3$, we construct $c_{0}^{1}=\overline{\mathcal{S}_{0}[Y, Y]}$, and, for $1 \leq k \leq n-1$, we set:

$$
\begin{aligned}
& c_{k}^{3}=\left[\bigcup_{t=0}^{(n-1) / 3-1}\left(\mathcal{D}_{k}[Y, X]\left\{\star x_{C_{k}^{3 t+1}(1)} \star x_{C_{k}^{3 t+2}(1)}\right\} \cup \mathcal{D}_{k}[X, Y]\left\{x_{C_{k}^{3 t+2}(1)} \star x_{C_{k}^{3 t+3}(1)} \star\right\}\right)\right] \cup \\
& {\left[\overline{\mathcal{S}_{k}[Y, Y]}\left\{\star x_{1} \star\right\}\right]} \\
& c_{k}^{4}=\left[\bigcup_{t=0}^{(n-1) / 3-1}\left(\mathcal{D}_{k}[Y, X]\left\{\star x_{C_{k}^{3 t+2}(1)} \star x_{C_{k}^{3 t+3}(1)}\right\} \cup \mathcal{D}_{k}[X, Y]\left\{x_{C_{k}^{3 t+3}(1)} \star x_{C_{k}^{3 t+4}(1)} \star\right\}\right)\right] \cup \\
& {\left[\overline{\mathcal{S}_{k}[Y, Y]}\left\{\star x_{C_{k}(1)} \star\right\}\right]} \\
& c_{k}^{5}=\left[\bigcup_{t=0}^{(n-1) / 3-1}\left(\mathcal{D}_{k}[Y, X]\left\{\star x_{C_{k}^{3 t+3}(1)} \star x_{C_{k}^{3 t+4}(1)}\right\} \cup \mathcal{D}_{k}[X, Y]\left\{x_{C_{k}^{3 t+4}(1)} \star x_{C_{k}^{3 t+5}(1)} \star\right\}\right)\right] \cup \\
& {\left[\overline{\mathcal{S}_{k}[Y, Y]}\left\{\star x_{C_{k}^{2}(1)} \star\right\}\right]} \\
& c_{k}^{1}=\left[\left(\overline{\mathcal{S}_{k}[Y, Y]}\right) \backslash\left(\overline{\mathcal{S}_{k}[Y, Y]}\left\{\star x_{1} \star\right\} \cup \overline{\mathcal{S}_{k}[Y, Y]}\left\{\star x_{C_{k}(1)} \star\right\} \cup \overline{\mathcal{S}_{k}[Y, Y]}\left\{\star x_{C_{k}^{2}(1)} \star\right\}\right)\right] \cup
\end{aligned}
$$




$$
\left[\mathcal{D}_{k}[Y, X]\left\{\star x_{1} \star x_{C_{k}(1)}\right\} \cup \mathcal{D}_{k}[X, Y]\left\{x_{C_{k}(1)} \star x_{C_{k}^{2}(1)} \star\right\}\right]
$$

As previously, Figure 5 shows the representation on $X$ of $c_{k}^{3}$, where the vertices of $X$ are sorted along $C_{k}$. A shift of one vertex on the right (modulo $n$ ) of the sets of paths gives the representation of $c_{k}^{4}$ for the same $k$. And a shift of two vertices gives the representation of $c_{k}^{5}$.

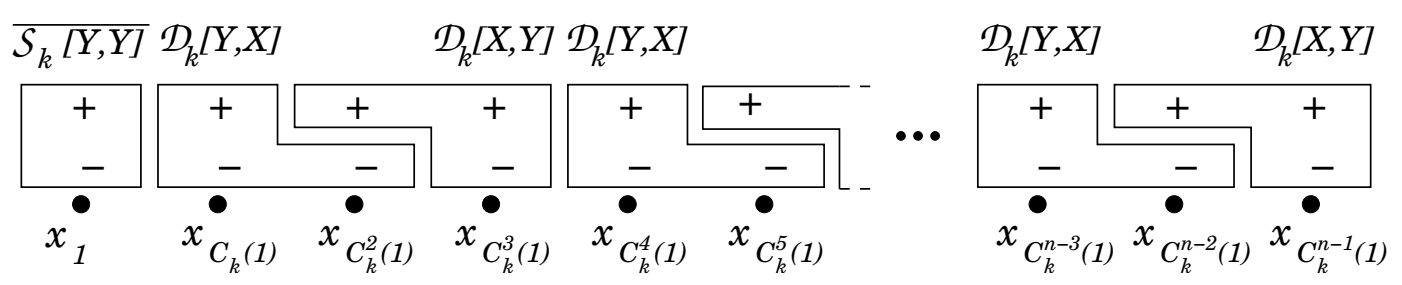

Figure 5: The representation on $X$ of the color class $c_{k}^{3}$, where the vertices of $X$ are sorted along $C_{k}$.

Figure 6 gives the representation on $X$ of the color classes $c_{k}^{1}$. Note that $c_{k}^{1}$ is well defined only if $n \geq 3$.

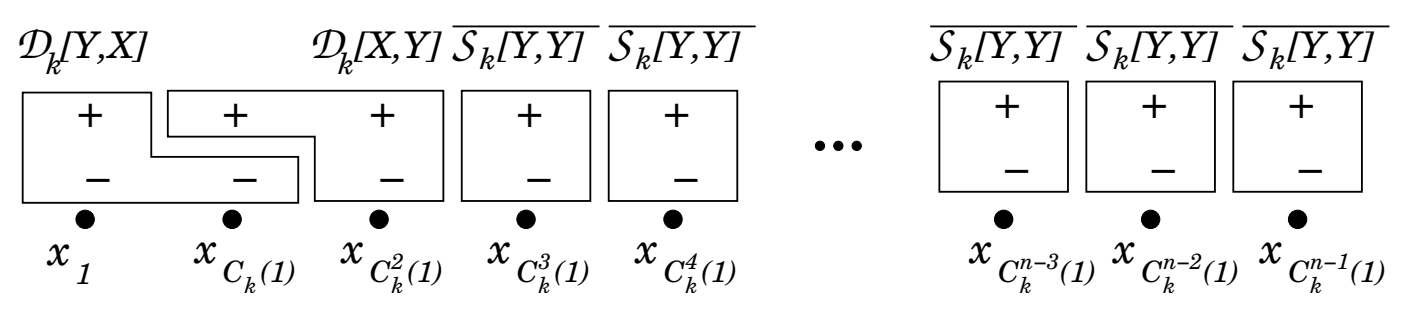

Figure 6: The representation on $X$ of the color class $c_{k}^{1}$, where the vertices of $X$ are sorted along $C_{k}$.

Now, the exact optical index will be obtained by packing the remaining paths of length 1 and 2, as done previously: the classes $c_{k}^{2}$ for $0 \leq k \leq f$ are defined exactly as in the case $n \equiv 0$ (mod 3). Indeed, even if the definitions of classes $c_{k}^{1}, c_{k}^{3}, c_{k}^{4}$ and $c_{k}^{5}$ have changed, the use of the paths $\mathcal{D}_{0}\left\{M_{i}\right\}$ is still the same: for every $k, 0 \leq k \leq f$, we have $\mathcal{D}_{0}\left\{M_{k}\right\} \subset c_{k}^{1} \cup c_{k}^{3} \cup c_{k}^{4} \cup c_{k}^{5}$. Finally, we obtain:

- If $1 \leq f \leq\lceil n / 2\rceil-2, w_{f}\left(K_{n, n}^{\star}\right)=5 f+3$.

- If $\lceil n / 2\rceil-1 \leq f \leq n-2, w_{f}\left(K_{n, n}^{\star}\right)=5 f+2$. 
- And, if $f=n-1, w_{f}\left(K_{n, n}^{\star}\right)=5 f+1$.

\subsection{Optical index in case $n \equiv 2(\bmod 3)$}

We proceed as in case $n \equiv 1(\bmod 3)$, except that this time we supplement each class of paths of length 3 with two sets of paths from $\overline{\mathcal{S}_{k}[Y, Y]}$. Conversely, the classes $c_{k}^{1}$ contain the remaining paths of $\overline{\mathcal{S}_{k}[Y, Y]}$ and 4 sets of paths of length 3 from $\mathcal{D}_{k}$, provided that $n \geq 6$. Precisely, for $n \geq 6$, we construct $c_{0}^{1}=\overline{\mathcal{S}_{0}[Y, Y]}$, and for $1 \leq k \leq n-1$, we set:

$$
\begin{aligned}
& c_{k}^{3}=\left[\bigcup_{t=0}^{(n-2) / 3-1}\left(\mathcal{D}_{k}[Y, X]\left\{\star x_{C_{k}^{3 t+2}(1)} \star x_{C_{k}^{3 t+3}(1)}\right\} \cup \mathcal{D}_{k}[X, Y]\left\{x_{C_{k}^{3 t+3}(1)} \star x_{C_{k}^{3 t+4}(1)} \star\right\}\right)\right] \cup \\
& {\left[\overline{\mathcal{S}_{k}[Y, Y]}\left\{\star x_{1} \star\right\} \cup \overline{\mathcal{S}_{k}[Y, Y]}\left\{\star x_{C_{k}(1)} \star\right\}\right]} \\
& c_{k}^{4}=\left[\bigcup_{t=0}^{(n-2) / 3-1}\left(\mathcal{D}_{k}[Y, X]\left\{\star x_{C_{k}^{3 t+4}(1)} \star x_{C_{k}^{3 t+5}(1)}\right\} \cup \mathcal{D}_{k}[X, Y]\left\{x_{C_{k}^{3 t+5}(1)} \star x_{C_{k}^{3 t+6}(1)} \star\right\}\right)\right] \cup \\
& {\left[\overline{\mathcal{S}_{k}[Y, Y]}\left\{\star x_{C_{k}^{2}(1)} \star\right\} \cup \overline{\mathcal{S}_{k}[Y, Y]}\left\{\star x_{C_{k}^{3}(1)} \star\right\}\right]} \\
& c_{k}^{5}=\left[\bigcup_{t=0}^{(n-2) / 3-1}\left(\mathcal{D}_{k}[Y, X]\left\{\star x_{C_{k}^{3 t+6}(1)} \star x_{C_{k}^{3 t+7}(1)}\right\} \cup \mathcal{D}_{k}[X, Y]\left\{x_{C_{k}^{3 t+7}(1)} \star x_{C_{k}^{3 t+8}(1)} \star\right\}\right)\right] \cup \\
& {\left[\overline{\mathcal{S}_{k}[Y, Y]}\left\{\star x_{C_{k}^{4}(1)} \star\right\} \cup \overline{\mathcal{S}_{k}[Y, Y]}\left\{\star x_{C_{k}^{5}(1)} \star\right\}\right]} \\
& c_{k}^{1}=\left[( \overline { \mathcal { S } _ { k } [ Y , Y ] } ) \backslash \left(\overline{\mathcal{S}_{k}[Y, Y]}\left\{\star x_{1} \star\right\} \cup \overline{\mathcal{S}_{k}[Y, Y]}\left\{\star x_{C_{k}(1)} \star\right\} \cup \overline{\mathcal{S}_{k}[Y, Y]}\left\{\star x_{C_{k}^{2}(1)} \star\right\} \cup\right.\right. \\
& \left.\left.\overline{\mathcal{S}_{k}[Y, Y]}\left\{\star x_{C_{k}^{3}(1)} \star\right\} \cup \overline{\mathcal{S}_{k}[Y, Y]}\left\{\star x_{C_{k}^{4}(1)} \star\right\} \cup \overline{\mathcal{S}_{k}[Y, Y]}\left\{\star x_{C_{k}^{5}(1)} \star\right\}\right)\right] \cup \\
& {\left[\mathcal{D}_{k}[Y, X]\left\{\star x_{1} \star x_{C_{k}(1)}\right\} \cup \mathcal{D}_{k}[X, Y]\left\{x_{C_{k}(1)} \star x_{C_{k}^{2}(1)} \star\right\} \cup\right.} \\
& \left.\mathcal{D}_{k}[Y, X]\left\{\star x_{C_{k}^{3}}(1) \star x_{C_{k}^{4}(1)}\right\} \cup \mathcal{D}_{k}[X, Y]\left\{x_{C_{k}^{4}(1)} \star x_{C_{k}^{5}(1)} \star\right\}\right]
\end{aligned}
$$


Once again, we give in Figure 7 the representation on $X$ of $c_{k}^{3}$, assuming that vertices of $X$ are sorted along $C_{k}$. A shift of two vertices on the right side (modulo $n$ ) of the sets of paths gives the representation of $c_{k}^{4}$, for the same $k$. And a shift of four vertices gives the representation of $c_{k}^{5}$

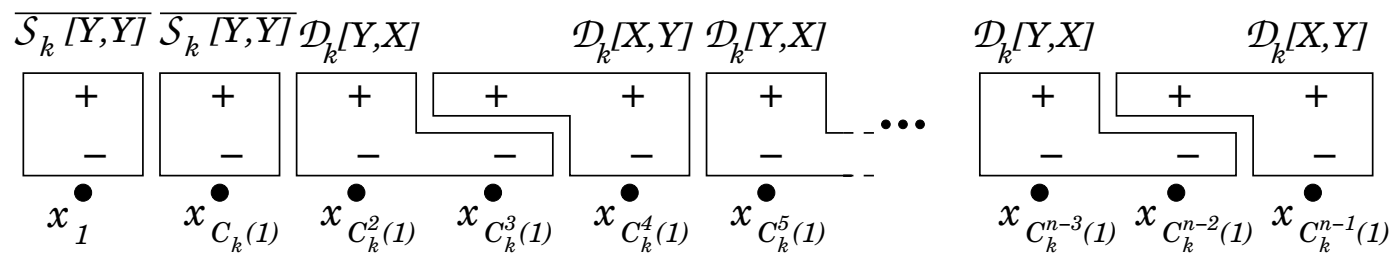

Figure 7: The representation on $X$ of the color class $c_{k}^{3}$, where the vertices of $X$ are sorted along $C_{k}$.

Figure 8 gives the representation on $X$ of the color classes $c_{k}^{1}$. Note that $c_{k}^{1}$ is well defined only if $n \geq 6$.

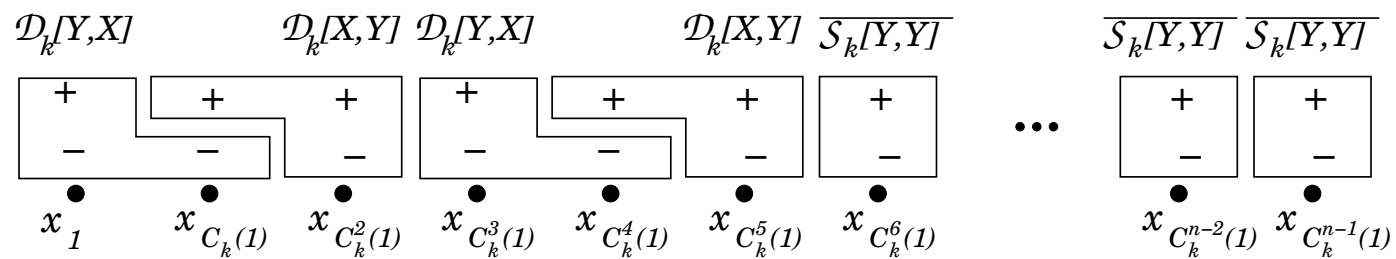

Figure 8: The representation on $X$ of the color class $c_{k}^{1}$, where the vertices of $X$ are sorted along $C_{k}$.

To conclude, the classes $c_{k}^{2}$ for $0 \leq k \leq f$ are defined exactly as in the cases $n \equiv 0(\bmod 3)$ and $n \equiv 1(\bmod 3)$. Once again, the paths $\mathcal{D}_{0}\left\{M_{k}\right\}$ are used as previously, and we obtain:

- If $1 \leq f \leq\lceil n / 2\rceil-2, w_{f}\left(K_{n, n}^{\star}\right)=5 f+3$.

- If $\lceil n / 2\rceil-1 \leq f \leq n-2, w_{f}\left(K_{n, n}^{\star}\right)=5 f+2$.

- And, if $f=n-1, w_{f}\left(K_{n, n}^{\star}\right)=5 f+1$. 


\subsection{Remaining particular cases}

As previously noted, five cases were not treated in the previous study: $n=2$ because of the non-existence of a Latin square of order $2, n=4$ and $n=6$ due to the exception to Tillson's Theorem and $n=1$ and $n=5$, where the general techniques for $n=1 \bmod 3$ and $n=2$ mod 3 fail. For all of these cases, we provide routings that give, for every $f, 0 \leq f \leq n-1$, $w_{f}\left(K_{n, n}^{\star}\right)=\pi_{f}\left(K_{n, n}^{\star}\right)$. These cases illustrate the above-mentioned general method and are studied in an appendix, available at: http://www.lirmm.fr/ bessy/publis.html.

\section{Acknowledgment}

We thank Jean-Claude Bermond for introducing the problem to us and for motivating our research.

\section{References}

[1] B. Beauquier, J.-C. Bermond, L. Gargano, P. Hell, S. Pérennes, and U. Vaccaro, Graph problems arising from wavelength-routing in all-optical networks, INRIA technical report 3165, May 1997 (presented at the Second IEEE Workshop on Optics and Computer Science).

[2] B. Beauquier, P. Hell, and S. Pérennes, Optimal wavelength-routed multicasting, Discrete Applied Mathematics 84 (1998), 15-20

[3] R. Diestel, Graph theory, Third edition, Graduate Texts in Mathematics, 173, SpringerVerlag, Berlin, 2005.

[4] J.H. Dinitz, A.C.H. Ling, and D.R. Stinson, Fault tolerant routings with minimum optical index, Networks 48 (2006), 47-54.

[5] A. Gupta, J. Maňuch, and L. Stacho, Fault tolerant forwarding and optical indexes: a design theory approach, Journal of Combinatorial Designs 14 (2006), 25-40.

[6] C.C. Lindner, and C.A. Rodger, Design theory, CRC Press, Inc. Boca Raton, FL, 1997. 
[7] J. Maňuch, and L. Stacho, On $f$-wise arc forwarding index and wavelength allocations in faulty all-optical hypercubes, Theoretical Informatics and Applications 37 (2003), 255-270.

[8] T.W. Tillson, A Hamiltonian decomposition of $K_{2 m}^{\star}, 2 m \geq 8$, J. Combin. Theory Ser. B 2 (1980), 68-74.

[9] V.G. Vizing, On an estimate of the chromatic class of a p-graph, Diskret. Analiz 3 (1964), $25-30$. 


\section{Appendix}

\section{A Routing in $K_{1,1}^{\star}$}

We are in the case $n \equiv 1(\bmod 3)$, but the general decomposition is valid for $n \geq 3$. However, here, there is just one value of $f$ to study: $f=0$ and the direct routing immediately gives $w_{0}\left(K_{1,1}^{\star}\right)=\pi_{0}\left(K_{1,1}^{\star}\right)=1$.

\section{B Routing in $K_{2,2}^{\star}$}

For $n=2$, there is no idempotent Latin square of order 2 , so we provide precisely the routing.

- For $f=0, \mathcal{D}_{0}$ forms a color class, and we choose the set of pairwise arc-disjoint paths of length 2 given by $\mathcal{S}_{0}=\left\{x_{1} y_{1} x_{2}, x_{2} y_{2} x_{1}, y_{1} x_{1} y_{2}, y_{2} x_{2} y_{1}\right\}$ to form the second color class. So, we obtain $w_{0}\left(K_{2,2}^{\star}\right)=\pi_{0}\left(K_{2,2}^{\star}\right)=2$.

- For $f=1$, we define $\mathcal{S}_{1}=\left\{x_{1} y_{2} x_{2}, x_{2} y_{1} x_{1}, y_{1} x_{2} y_{2}, y_{2} x_{1} y_{1}\right\}$, which contains pairwise arcdisjoint paths, and $\mathcal{D}_{1}=\left\{x_{i} y_{j+1} x_{i+1} y_{j}, y_{i} x_{j+1} y_{i+1} x_{j}: 1 \leq i \leq 2,1 \leq j \leq 2\right\}$ (which corresponds to usual definition of $\mathcal{D}_{1}$ ). The color classes are defined by: $c_{1}=\mathcal{S}_{0}, c_{2}=\mathcal{S}_{1}$ and $c_{(i, j)}=\mathcal{D}_{0}\left\{x_{i} y_{j}, y_{j} x_{i}\right\} \cup \mathcal{D}_{1}\left\{x_{i} y_{j+1} x_{i+1} y_{j}, y_{j} x_{i+1} y_{j+1} x_{i}\right\}$ for $1 \leq i \leq 2$ and $1 \leq j \leq 2$. Finally, we obtain $w_{1}\left(K_{2,2}^{\star}\right)=\pi_{1}\left(K_{2,2}^{\star}\right)=6$.

\section{Routing in $K_{4,4}^{\star}$}

The case $n=4$ is the first exception to Tillson's Theorem of decomposition, so we define in Figure 9 the partition of the arcs of $K_{4}^{\star}$. Then, for the different values of $f$, the routings are defined as previously, in Section 4.1.

- For $f=0, f=1$, and $f=2$, since $C_{1}$ and $C_{2}$ are disjoint Hamiltonian circuits of $K_{4}^{\star}$, the routings are defined exactly as in the general case $n \equiv 1(\bmod 3)$. So, in these cases, we obtain $w_{f}\left(K_{4}^{\star}\right)=\pi_{f}\left(K_{4}^{\star}\right)$.

- For $f=3$, we need to pack the paths of $\mathcal{R}_{3}$. We differently organize the arcs of $K_{4}^{\star}$ in order to obtain a suitable decomposition of the paths. The scheme of the routing is given Figure 10. The arcs are labeled with the name of the circuit $C_{i}$ they belong to. 


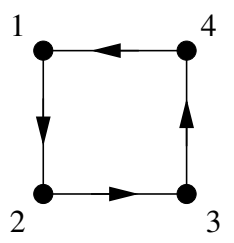

$C_{1}$

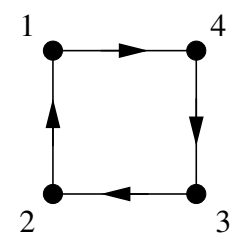

$C_{2}$

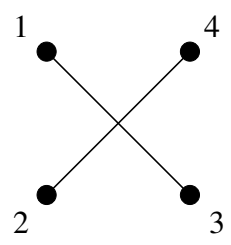

$C_{3}$

Figure 9: The chosen decomposition of the $\operatorname{arcs}$ of $K_{4}^{\star}$.
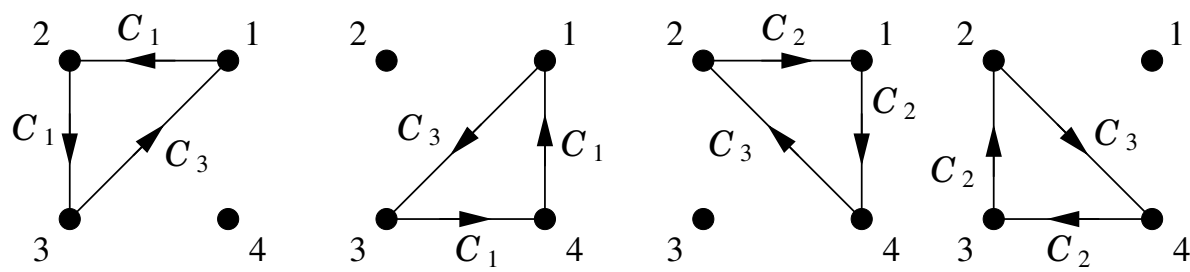

Figure 10: New decomposition of the $\operatorname{arcs}$ of $K_{4}^{\star}$ in case $f=3$.

Now, using these circuits, we route almost as in general case $n \equiv 1(\bmod 3)$. Using paths of some $\mathcal{D}_{k}$ and $\overline{\mathcal{S}_{k}[Y, Y]}$, each one of these new circuits gives three color classes. The remaining paths, mainly from $\mathcal{S}_{k}[X, X]$, are packed together to form the four remaining colors.

More precisely, the first circuit provides the following classes:

$$
\begin{aligned}
& c_{1}=\mathcal{D}_{1}[Y, X]\left\{\star x_{1} \star x_{2}\right\} \cup \mathcal{D}_{1}[X, Y]\left\{x_{2} \star x_{3} \star\right\} \cup \overline{\mathcal{S}_{1}[Y, Y]}\left\{\star x_{4} \star\right\} \\
& c_{2}=\mathcal{D}_{1}[Y, X]\left\{\star x_{2} \star x_{3}\right\} \cup \mathcal{D}_{3}[X, Y]\left\{x_{3} \star x_{1} \star\right\} \cup \overline{\mathcal{S}_{2}[Y, Y]}\left\{\star x_{4} \star\right\} \\
& c_{3}=\mathcal{D}_{3}[Y, X]\left\{\star x_{3} \star x_{1}\right\} \cup \mathcal{D}_{1}[X, Y]\left\{x_{1} \star x_{2} \star\right\} \cup \overline{\mathcal{S}_{3}[Y, Y]}\left\{\star x_{4} \star\right\}
\end{aligned}
$$

From the second circuit, we obtain:

$$
\begin{aligned}
& c_{4}=\mathcal{D}_{3}[Y, X]\left\{\star x_{1} \star x_{3}\right\} \cup \mathcal{D}_{1}[X, Y]\left\{x_{3} \star x_{4} \star\right\} \cup \overline{\mathcal{S}_{1}[Y, Y]}\left\{\star x_{2} \star\right\} \\
& c_{5}=\mathcal{D}_{1}[Y, X]\left\{\star x_{3} \star x_{4}\right\} \cup \mathcal{D}_{1}[X, Y]\left\{x_{4} \star x_{1} \star\right\} \cup \overline{\mathcal{S}_{2}[Y, Y]}\left\{\star x_{2} \star\right\} \\
& c_{6}=\mathcal{D}_{1}[Y, X]\left\{\star x_{4} \star x_{1}\right\} \cup \mathcal{D}_{3}[X, Y]\left\{x_{1} \star x_{3} \star\right\} \cup \overline{\mathcal{S}_{3}[Y, Y]}\left\{\star x_{2} \star\right\}
\end{aligned}
$$

From the third circuit, we obtain:

$$
c_{7}=\mathcal{D}_{2}[Y, X]\left\{\star x_{1} \star x_{4}\right\} \cup \mathcal{D}_{3}[X, Y]\left\{x_{4} \star x_{2} \star\right\} \cup \overline{\mathcal{S}_{1}[Y, Y]}\left\{\star x_{3} \star\right\}
$$




$$
\begin{aligned}
& c_{8}=\mathcal{D}_{3}[Y, X]\left\{\star x_{4} \star x_{2}\right\} \cup \mathcal{D}_{2}[X, Y]\left\{x_{2} \star x_{1} \star\right\} \cup \overline{\mathcal{S}_{2}[Y, Y]}\left\{\star x_{3} \star\right\} \\
& c_{9}=\mathcal{D}_{2}[Y, X]\left\{\star x_{2} \star x_{1}\right\} \cup \mathcal{D}_{2}[X, Y]\left\{x_{1} \star x_{4} \star\right\} \cup \overline{\mathcal{S}_{3}[Y, Y]}\left\{\star x_{3} \star\right\}
\end{aligned}
$$

Finally, we obtain from the fourth circuit:

$$
\begin{aligned}
& c_{10}=\mathcal{D}_{3}[Y, X]\left\{\star x_{2} \star x_{4}\right\} \cup \mathcal{D}_{2}[X, Y]\left\{x_{4} \star x_{3} \star\right\} \cup \overline{\mathcal{S}_{1}[Y, Y]}\left\{\star x_{1} \star\right\} \\
& c_{11}=\mathcal{D}_{2}[Y, X]\left\{\star x_{4} \star x_{3}\right\} \cup \mathcal{D}_{2}[X, Y]\left\{x_{3} \star x_{2} \star\right\} \cup \overline{\mathcal{S}_{2}[Y, Y]}\left\{\star x_{1} \star\right\} \\
& c_{12}=\mathcal{D}_{2}[Y, X]\left\{\star x_{3} \star x_{2}\right\} \cup \mathcal{D}_{3}[X, Y]\left\{x_{2} \star x_{4} \star\right\} \cup \overline{\mathcal{S}_{3}[Y, Y]}\left\{\star x_{1} \star\right\}
\end{aligned}
$$

The next class uses $\mathcal{S}_{0}[Y, Y]$ and the last matching of $\mathcal{D}_{0}$ :

$$
c_{13}=\overline{\mathcal{S}_{0}[Y, Y]}
$$

To conclude, as previously in the case $n \equiv 0(\bmod 3)$, we pack the paths of $\bigcup_{k=0}^{3} \mathcal{S}_{k}[X, X]$ in three color classes:

$$
\begin{aligned}
& c_{14}=\bigcup_{j=1}^{4} \bigcup_{i=0}^{3} \mathcal{S}_{i}[X, X]\left\{x_{j} \star x_{j+1}\right\} \\
& c_{15}=\bigcup_{j=1}^{4} \bigcup_{i=0}^{3} \mathcal{S}_{i}[X, X]\left\{x_{j} \star x_{j+2}\right\} \\
& c_{16}=\bigcup_{j=1}^{4} \bigcup_{i=0}^{3} \mathcal{S}_{i}[X, X]\left\{x_{j} \star x_{j+3}\right\}
\end{aligned}
$$

Finally, we obtain $w_{3}\left(K_{4,4}^{\star}\right)=\pi_{3}\left(K_{4,4}^{\star}\right)=16$.

\section{Routing in $K_{5,5}^{\star}$}

We are in the case $n \equiv 2(\bmod 3)$, but the general decomposition is only valid for $n \geq 6$. Indeed, for every $k$, we need six sets of $\overline{\mathcal{S}_{k}[Y, Y]}$ centered on six different vertices to complete the three color classes constructed from the paths of $\mathcal{D}_{k}$. In the case $n=5$, we need to use paths from different sets $\overline{\mathcal{S}_{k}[Y, Y]}$ to complete the color classes constructed from a single set $\mathcal{D}_{k}$.

To simplify the notation, we fix, in Figure 11, a decomposition of the $\operatorname{arcs}$ of $K_{5}^{\star}$. Using this decomposition we define the routing sets as previously, in Section 4.1.

Now, we detail how to pack the paths for all values of $f$. 

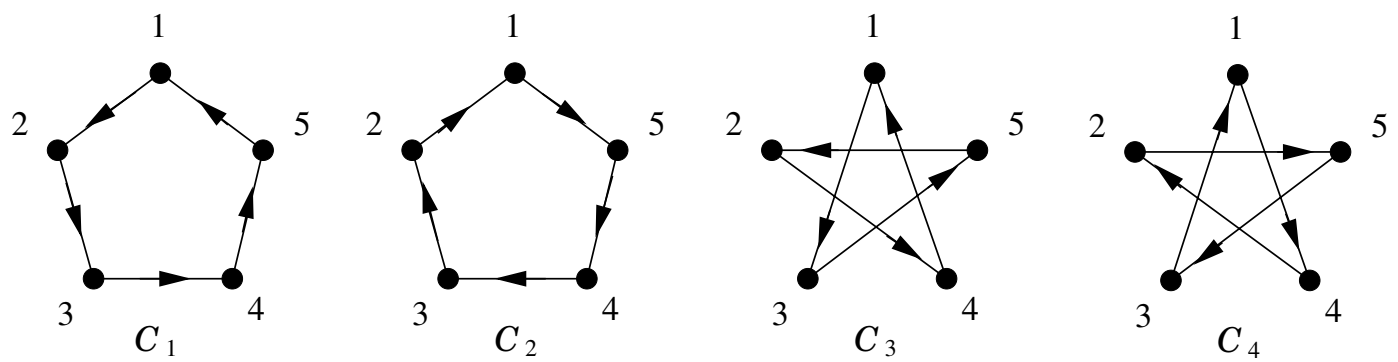

Figure 11: An explicit decomposition of the arcs of $K_{5}^{\star}$.

- For $f=0$, as usual, we set $c_{0}^{1}=\mathcal{D}_{0}, c_{0}^{2}=\mathcal{S}_{0}[X, X]$ and $c_{0}^{3}=\mathcal{S}_{0}[Y, Y]$ and have $w_{0}\left(K_{5,5}^{\star}\right)=$ $\pi_{0}\left(K_{5,5}^{\star}\right)=3$.

- For $f=1$, we use paths from $\overline{\mathcal{S}_{0}[Y, Y]}$ and $\overline{\mathcal{S}_{1}[Y, Y]}$ to complete the colors obtained with paths from $\mathcal{D}_{1}$. More precisely, we define for $k=1, \ldots, 5$ :

$$
\begin{gathered}
c_{1}^{k}=\mathcal{D}_{1}[Y, X]\left\{\star x_{k} \star x_{k+1}\right\} \cup \mathcal{D}_{1}[X, Y]\left\{x_{k+1} \star x_{k+2} \star\right\} \cup \\
\overline{\mathcal{S}_{0}[Y, Y]}\left\{\star x_{k+3} \star\right\} \cup \overline{\mathcal{S}_{1}[Y, Y]}\left\{\star x_{k+4} \star\right\}
\end{gathered}
$$

The remaining paths form the three color classes $\mathcal{S}_{0}[X, X], \mathcal{S}_{1}[X, X]$ and $\mathcal{D}_{0}\left\{M_{0}, M_{1}, M_{2}\right\}$ $\left(\mathcal{D}_{0}\left\{M_{3}\right\}\right.$ and $\mathcal{D}_{0}\left\{M_{4}\right\}$ are respectively contained in $\overline{\mathcal{S}_{0}[Y, Y]}$ and $\overline{\mathcal{S}_{1}[Y, Y]}$ ). So, we obtain $w_{1}\left(K_{5,5}^{\star}\right)=\pi_{1}\left(K_{5,5}^{\star}\right)=8$.

- For $f=2$, the paths from $\mathcal{D}_{1}$ are packed as previously: for $k=1, \ldots, 5$ we use $c_{1}^{k}$. The paths from $\mathcal{D}_{2}$ form color classes with paths from $\overline{\mathcal{S}_{0}[X, X]}$ and $\overline{\mathcal{S}_{1}[X, X]}$. We define, for $k=1, \ldots, 5$ :

$$
\begin{gathered}
c_{2}^{k}=\mathcal{D}_{2}[X, Y]\left\{\star y_{k} \star y_{k-1}\right\} \cup \mathcal{D}_{2}[Y, X]\left\{y_{k-1} \star y_{k-2} \star\right\} \cup \\
\overline{\mathcal{S}_{0}[X, X]}\left\{\star y_{k-3} \star\right\} \cup \overline{\mathcal{S}_{1}[X, X]}\left\{\star y_{k-4} \star\right\}
\end{gathered}
$$

The remaining paths form the two color classes $\overline{\mathcal{S}_{2}[X, X]}$ and $\mathcal{S}_{2}[Y, Y]\left(\mathcal{D}_{0}\left\{M_{0}\right\}, \mathcal{D}_{0}\left\{M_{1}\right\}\right.$ and $\mathcal{D}_{0}\left\{M_{2}\right\}$ are respectively contained in $\overline{\mathcal{S}_{0}[X, X]}, \overline{\mathcal{S}_{1}[X, X]}$ and $\overline{\mathcal{S}_{2}[X, X]}$ ). So, we obtain $w_{2}\left(K_{5,5}^{\star}\right)=\pi_{2}\left(K_{5,5}^{\star}\right)=12$.

- For $f=3$, we use the same method to pack the paths from $\mathcal{D}_{3}$ with paths from $\mathcal{S}_{2}[Y, Y]$ and $\mathcal{S}_{3}[Y, Y]$. For the paths from $\mathcal{D}_{1}$ and $\mathcal{D}_{2}$, we use $c_{1}^{1}, \ldots, c_{1}^{5}$ and $c_{2}^{1}, \ldots, c_{2}^{5}$ and, for 
$k=1, \ldots, 5$, we define:

$$
\begin{gathered}
c_{3}^{k}=\mathcal{D}_{3}[Y, X]\left\{\star x_{k} \star x_{k+2}\right\} \cup \mathcal{D}_{3}[X, Y]\left\{x_{k+2} \star x_{k+4} \star\right\} \cup \\
\mathcal{S}_{2}[Y, Y]\left\{\star x_{k+1} \star\right\} \cup \mathcal{S}_{3}[Y, Y]\left\{\star x_{k+3} \star\right\}
\end{gathered}
$$

The remaining paths form the two color classes $\overline{\mathcal{S}_{2}[X, X]}$ and $\mathcal{S}_{3}[X, X]$ So, we obtain $w_{3}\left(K_{5,5}^{\star}\right)=\pi_{3}\left(K_{5,5}^{\star}\right)=17$.

- Finally, for $f=4$, we have to change color classes. Indeed, this time, paths from $\mathcal{D}_{i}$ are packed with sets of type $\left(\bigcup_{p=0}^{4} \mathcal{S}_{p}[X, X]\left\{x_{l} \star x_{m}\right\}\right) \cup\left(\bigcup_{p=0}^{4} \mathcal{S}_{p}[X, X]\left\{x_{m} \star x_{l}\right\}\right)$ which saturate exactly the in and out-neighborhood of the vertices $x_{l}$ and $x_{m}$. Precisely, we define, for $k=1, \ldots, 5$ :

$$
\begin{gathered}
c_{1}^{k}=\mathcal{D}_{1}[Y, X]\left\{\star x_{k} \star x_{k+1}\right\} \cup \mathcal{D}_{1}[X, Y]\left\{x_{k+1} \star x_{k+2} \star\right\} \cup \\
\left(\bigcup_{p=0}^{4} \mathcal{S}_{p}[X, X]\left\{x_{k+3} \star x_{k+4}\right\}\right) \cup\left(\bigcup_{p=0}^{4} \mathcal{S}_{p}[X, X]\left\{x_{k+4} \star x_{k+3}\right\}\right) \\
c_{2}^{k}=\mathcal{D}_{2}[X, Y]\left\{\star y_{k} \star y_{k-1}\right\} \cup \mathcal{D}_{2}[Y, X]\left\{y_{k-1} \star y_{k-2} \star\right\} \cup \\
\left(\bigcup_{p=0}^{4} \mathcal{S}_{p}[Y, Y]\left\{y_{k-3} \star y_{k-4}\right\}\right) \cup\left(\bigcup_{p=0}^{4} \mathcal{S}_{p}[Y, Y]\left\{y_{k-4} \star y_{k-3}\right\}\right) \\
c_{3}^{k}=\mathcal{D}_{3}[Y, X]\left\{\star x_{k} \star x_{k+2}\right\} \cup \mathcal{D}_{3}[X, Y]\left\{x_{k+2} \star x_{k+4} \star\right\} \cup \\
\left(\bigcup_{p=0}^{4} \mathcal{S}_{p}[X, X]\left\{x_{k+1} \star x_{k+3}\right\}\right) \cup\left(\bigcup_{p=0}^{4} \mathcal{S}_{p}[X, X]\left\{x_{k+3} \star x_{k+1}\right\}\right) \\
c_{4}^{k}=\mathcal{D}_{4}[X, Y]\left\{\star y_{k} \star y_{k-2}\right\} \cup \mathcal{D}_{4}[Y, X]\left\{y_{k-2} \star y_{k-4} \star\right\} \cup \\
\left(\bigcup_{p=0}^{4} \mathcal{S}_{p}[Y, Y]\left\{y_{k-1} \star y_{k-3}\right\}\right) \cup\left(\bigcup_{p=0}^{4} \mathcal{S}_{p}[Y, Y]\left\{y_{k-3} \star y_{k-1}\right\}\right)
\end{gathered}
$$

The remaining paths are exactly the direct paths, $\mathcal{D}_{0}$. So, we obtain $w_{4}\left(K_{5,5}^{\star}\right)=\pi_{4}\left(K_{5,5}^{\star}\right)=$ 21. 


\section{E Routing in $K_{6,6}^{\star}$}

The case $n=6$ is the second exception to Tillson's Theorem of decomposition, so we define in Figure 12 the partition of the arcs of $K_{6}^{\star}$. Then, for the different values of $f$, the routings are defined as previously, in Section 4.1.

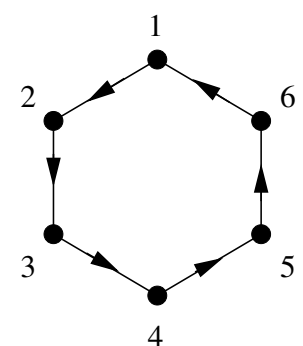

$C_{1}$

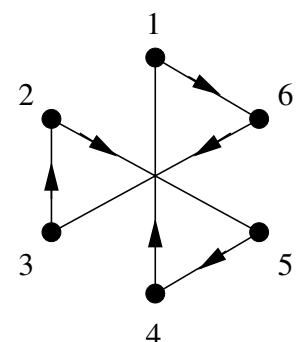

$\mathrm{C}_{2}$

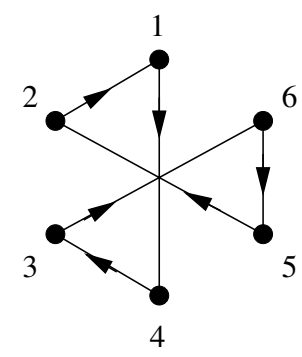

$\mathrm{C}_{3}$

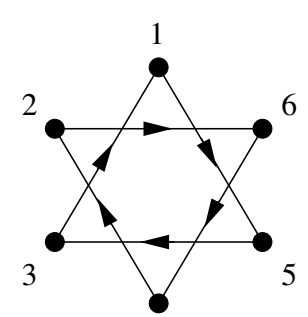

4

$\mathrm{C}_{4}$

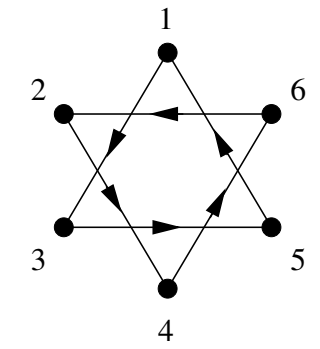

$C_{5}$

Figure 12: The chosen decomposition of the arcs of $K_{6}^{\star}$.

As all the circuits involved in this decomposition have length 3 or 6 , the computation of $w_{f}$ works exactly as in general case $n \equiv 0(\bmod 3)$. Finally, we obtain, for all $f=0, \ldots, 5$, $w_{f}\left(K_{6,6}^{\star}\right)=\pi_{f}\left(K_{6,6}^{\star}\right)$, which completes the proof of Theorem 4. 